\title{
$\mathrm{SU}(3)_{F}$ in nonleptonic charm decays*
}

\section{Gudrun Hiller}

Institut für Physik, Technische Universität Dortmund, D-44221 Dortmund, Germany

E-mail: gudrun.hiller@tu-dortmund.de

\section{Martin Jung}

Institut für Physik, Technische Universität Dortmund, D-44221 Dortmund, Germany

E-mail: martin2. jung@tu-dortmund.de

\section{Stefan Schacht ${ }^{\dagger}$}

Institut für Physik, Technische Universität Dortmund, D-44221 Dortmund, Germany Institut für Theoretische Teilchenphysik, Karlsruher Institut für Technologie, D-76128

Karlsruhe, Germany

E-mail: stefan.schachtakit.edu

We present updated results of an $\mathrm{SU}(3)$-flavor analysis of $D \rightarrow P P$ decay data including linear breaking and no further assumptions. The global fit is consistent with nominal $(\sim 30 \%) \mathrm{SU}(3)_{F}$ breaking and returns enhanced penguin (triplet) contributions. Their size is driven, in addition to $\Delta A_{C P}$, by the CP asymmetries of $D_{0} \rightarrow K_{S} K_{S}, D_{s} \rightarrow K_{S} \pi^{+}$and $D_{s} \rightarrow K^{+} \pi^{0}$ decays. It is therefore especially important to improve these measurements.

The European Physical Society Conference on High Energy Physics

18-24 July, 2013

Stockholm, Sweden

\footnotetext{
*DO-TH 13/30, QFET-2013-11, TTP13-038
}

† Speaker. 


\section{Introduction}

In 2011 and 2012, spectacular results indicated large CP violation in $D$ decays [1,2]. Before the Moriond conference in March 2013, the world average for the difference of the CP asymmetries $\Delta a_{C P}^{\mathrm{dir}}$ of $D^{0} \rightarrow K^{+} K^{-}$and $D^{0} \rightarrow \pi^{+} \pi^{-}$was $4.6 \sigma$ away from zero. This situation triggered a lot of theory papers $[3,4,5,6,7,8,9,10,11,12]$, especially trying to explain the effect in new physics models, e.g., $[13,14,15,16,17]$. Recently, LHCb updated the previous measurement of $\Delta A_{C P}$ and performed a further measurement in an additional channel, resulting in

$$
\begin{aligned}
& \Delta A_{C P}=-0.0034 \pm 0.0018, \quad \quad\left(D^{*} \text { decay channel [18] }\right) \\
& \Delta A_{C P}=0.0049 \pm 0.0033, \quad \text { (semileptonic } B \text { decay channel [19]) }
\end{aligned}
$$

where we added quadratically the statistical and systematic uncertainties. There is a tension of $2.2 \sigma[19]$ between both results, which differ in sign. The current world average including these results is $\Delta a_{C P}^{\mathrm{dir}}=-0.00333 \pm 0.00120$ [1, 2, 18, 19, 20, 21, 22], only $2.8 \sigma$ away from zero.

In the Standard Model (SM), one can write $\Delta a_{C P}^{\mathrm{dir}} \sim|P / T| \sin \delta \sin \gamma[6,23]$ with the penguin over tree ratio $P / T$, the CKM angle $\gamma$ and a strong phase $\delta$. With respect to the tree amplitude, the penguin one is CKM suppressed by $\lambda^{4} \sim 10^{-3}$ and a "naive" loop factor $\alpha_{s} / \pi \sim 0.1$. Altogether, this gives the rough estimate $\Delta a_{C P}^{\mathrm{dir}} \lesssim 10^{-4}$. The latter is however not reliable because $\alpha_{s}\left(m_{c}\right)$ is large and the expansion in $\Lambda_{\mathrm{QCD}} / m_{c}$ is not expected to converge fast. Consequently, it is unclear if a large $\Delta A_{C P}$ indicates new physics or is a QCD effect. In order to approach this problem we include additional observables which we relate by a symmetry principle.

\section{2. $\mathrm{SU}(3)_{F}$ approach to nonleptonic $D$ decays}

Besides $\Delta a_{C P}^{\text {dir }}$ many other observables of $D \rightarrow P P$ decays have been measured. It is important to obtain a complete picture of all singly Cabibbo suppressed (SCS) CP asymmetries as well as SCS, Cabibbo-favored (CF) and doubly Cabibbo suppressed (DCS) branching ratios. In a datadriven way we use the approximate $\mathrm{SU}(3)_{F}$ symmetry of QCD including linear breaking to relate several decay modes of $D \rightarrow P P$. For that, we analyze the operators of the effective Hamiltonian as well as initial and final states on their $\mathrm{SU}(3)_{F}$ representations. Subsequently, we use the WignerEckart theorem in order to obtain the corresponding reduced matrix elements, all of which we fit from data only. The amplitudes of SCS, CF and DCS amplitudes can then be written as

$$
\begin{aligned}
& \mathscr{A}(d)=\Sigma\left(\sum_{i, k} c_{d ; i k} A_{i}^{k}+\sum_{i, j} c_{d ; i j} B_{i}^{j}\right) \\
& \mathscr{A}(d)=V_{c s}^{*} V_{u d}\left(\sum_{i, k} c_{d ; i k} A_{i}^{k}+\sum_{i, j} c_{d ; i j} B_{i}^{j}\right), \\
& \mathscr{A}(d)=V_{c d}^{*} V_{u s}\left(\sum_{i, k} c_{d ; i k} A_{i}^{k}+\sum_{i, j} c_{d ; i j} B_{i}^{j}\right),
\end{aligned}
$$

with $\Sigma \equiv\left(V_{c s}^{*} V_{u s}-V_{c d}^{*} V_{u d}\right) / 2$, the $\mathrm{SU}(3)_{F}$ limit matrix elements $A_{i}^{k}$, the $\mathrm{SU}(3)_{F}$-breaking matrix elements $B_{i}^{j}$ and Clebsch-Gordan coefficients $c_{d ; i k}$. CP violation is induced by the interference of 


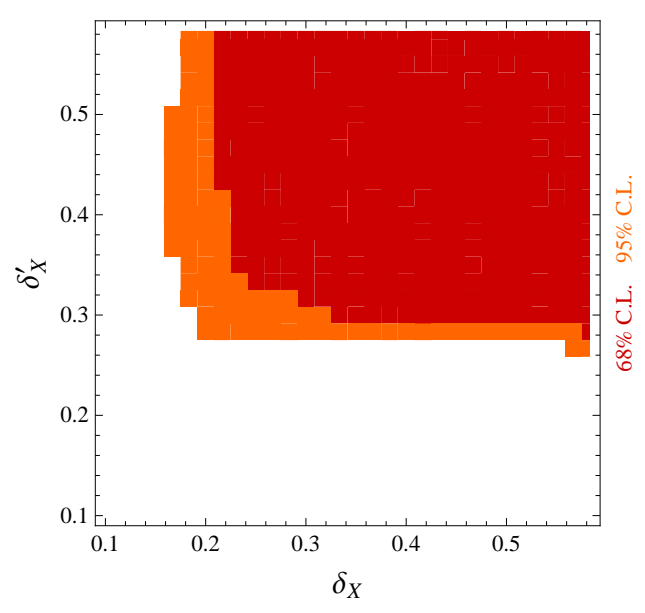

(a) $\mathrm{SU}(3)_{F}$ breaking.

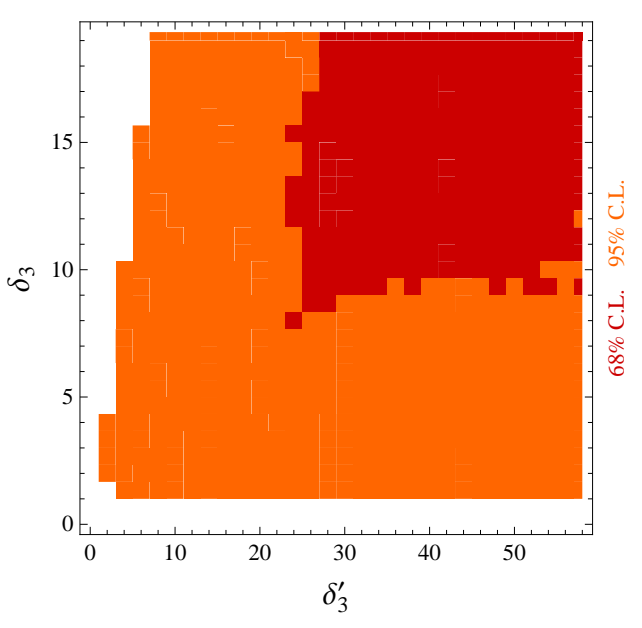

(b) Relative size of Penguins.

Figure 1: Fit results for $\mathrm{SU}(3)_{F}$ breaking and penguin enhancement, taking all present data given in Table II of [24] and updated in Table 1 into account. The red (orange) regions are allowed at $68 \%$ and 95\% C.L., respectively, see text for details. Figures taken from [25].

terms coming with the CKM factors $\Sigma$ and $\Delta \equiv\left(V_{c s}^{*} V_{u s}+V_{c d}^{*} V_{u d}\right) / 2$. The dominant contribution to CP-violating observables is coming from penguin matrix elements $A_{1}^{3}$ and $A_{8}^{3}$ that come with coefficients $c_{d ;(1,8) 3} \propto \Delta$. The SU(3) $F$ decomposition and the corresponding Clebsch-Gordan coefficients can be found in [24].

Here we present, as part of recent work [25], an update of our SU(3) $)_{F}$ study [24] of the presently available data on two-body charm decays to kaons and pions. Since fall of 2012, several new measurements appeared, which modify the world averages of the CP asymmetries $\Delta a_{C P}^{\mathrm{dir}}, \Sigma a_{C P}^{\mathrm{dir}} \equiv$ $a_{C P}^{\mathrm{dir}}\left(D^{0} \rightarrow K^{+} K^{-}\right)+a_{C P}^{\mathrm{dir}}\left(D^{0} \rightarrow \pi^{+} \pi^{-}\right), a_{C P}^{\mathrm{dir}}\left(D_{s} \rightarrow K_{S} \pi^{+}\right)$, the strong phase $\delta_{K \pi}$ as well as the branching ratio of the CF decay $D_{s} \rightarrow K_{S} K^{+}$. For a comparison with the values used in [24] as well as detailed references see Table 1. Besides the decrease of $\Delta a_{C P}^{\mathrm{dir}}$ and $a_{C P}^{\mathrm{dir}}\left(D_{s} \rightarrow K_{S} \pi^{+}\right)$, the measurement of $\Sigma a_{C P}^{\mathrm{dir}}$ gained precision and its central value approached zero. The latter is in accord with the $U$-spin limit relation $\Sigma a_{C P}^{\mathrm{dir}}=0$.

The fits shown are performed using the NLopt code [26] with the algorithms that can be found in $[26,27,28,29]$.

\section{Extraction of $\mathrm{SU}(3)_{F}$ breaking and penguins from present data}

In order to quantify the $\mathrm{SU}(3)_{F}$ breaking and the size of the penguins we introduce the following complementary measures [24]:

$$
\begin{array}{ll}
\delta_{X}=\frac{\max _{i j}\left|B_{i}^{j}\right|}{\max \left(\left|A_{27}^{15}\right|,\left|A_{8}^{\overline{6}}\right|,\left|A_{8}^{15}\right|\right)}, \quad \delta_{X}^{\prime}=\max _{d}\left|\frac{\mathscr{A}_{X}(d)}{\mathscr{A}(d)}\right| \\
\delta_{3}=\frac{\max \left(\left|A_{1}^{3}\right|,\left|A_{8}^{3}\right|\right)}{\max \left(\left|A_{27}^{15}\right|,\left|A_{8}^{\overline{6}}\right|,\left|A_{8}^{15}\right|\right)}, \quad \delta_{3}^{\prime}=\max _{d}\left|\frac{c_{d ; 13} A_{1}^{3}+c_{d ; 83} A_{8}^{3}}{c_{d ; 2715} A_{27}^{15}+c_{d ; 8 \overline{6}} A_{8}^{\overline{6}}+c_{d ; 8} A_{8}^{15}}\right| .
\end{array}
$$




\begin{tabular}{cccr}
\hline \hline Observable & Average Nov 2013 & Average Nov 2012 [24] & References \\
\hline \hline$\Delta a_{C P}^{\mathrm{dir}}\left(K^{+} K^{-}, \pi^{+} \pi^{-}\right)$ & $-0.00333 \pm 0.00120$ & $-0.00678 \pm 0.00147$ & {$[20],[1,2,18,19]$} \\
& & & {$[21,22,30,31]$} \\
$\Sigma a_{C P}^{\mathrm{dir}}\left(K^{+} K^{-}, \pi^{+} \pi^{-}\right)$ & $+0.00008 \pm 0.00228$ & $+0.0014 \pm 0.0039$ & $\dagger[1,2,22,30,32]$ \\
$a_{C P}^{\mathrm{dir}}\left(D_{s} \rightarrow K_{S} \pi^{+}\right)$ & $+0.011 \pm 0.007$ & $+0.028 \pm 0.015$ & $\dagger[33,34,35,36]$ \\
$a_{C P}^{\text {ind }}$ & $+0.00015 \pm 0.00052$ & $-0.00027 \pm 0.00163$ & {$[20]$} \\
$\delta_{K \pi}$ & $(11.7 \pm 10.2)^{\circ}$ & $(21.4 \pm 10.4)^{\circ}$ & ${ }^{\dagger}[20]$ \\
$\mathscr{B}\left(D_{s} \rightarrow K_{S} K^{+}\right)$ & $(1.50 \pm 0.05) \cdot 10^{-2}$ & $(1.45 \pm 0.05) \cdot 10^{-2}$ & $\dagger[37,38]$ \\
\hline \hline
\end{tabular}

Table 1: Updates of averages of experimental measurements compared to the status in Nov 2012 given in [24]. 'Our average where we added systematic and statistical error quadratically. ${ }^{\dagger}$ Uncertainties calculated by symmetrization. Table adapted from [24] and [25].

Here, $\mathscr{A}_{X}(d)$ is the $\mathrm{SU}(3)_{F}$-breaking part of the amplitudes, i.e., $\mathscr{A}(d)$ with $A_{i}^{k}=0$. In the definition of the measures $\delta_{X}^{\prime}$ and $\delta_{3}^{\prime}$ we do not take into account the decay $D^{0} \rightarrow K_{S} K_{S}$. The latter would introduce a bias, since, in contrast to all other considered decays, its $\mathrm{SU}(3)_{F}$ limit is CKMsuppressed $\propto \Delta$. The ratios of $\mathrm{SU}(3)_{F}$ matrix elements $\delta_{X}$ and $\delta_{3}$ do not take into account effects of small Clebsch-Gordan coefficients in front of the respective matrix elements. On the other hand, the ratios of parts of amplitudes, $\delta_{X}^{\prime}$ and $\delta_{3}^{\prime}$ do not take into account possible large cancellations between different summands. It is therefore essential to study both measures to achieve the full information on the $\mathrm{SU}(3)_{F}$ breaking and the penguin enhancement.

The truncation of the perturbative $\mathrm{SU}(3)_{F}$ expansion at the next to leading order is only sensible if the $\mathrm{SU}(3)_{F}$ breaking is not too large. Therefore at first we validate our ansatz. In Fig. 1(a), we show our fit results for the $\mathrm{SU}(3)_{F}$ breaking with present data as summarized in Table II of [24] and updated in Table 1. Already an $\mathrm{SU}(3)_{F}$ breaking of $\sim 30 \%$ can describe the data reasonably well, while larger $\mathrm{SU}(3)_{F}$ breaking cannot be excluded from data only. This is true independent of the measure $\delta_{X}$ or $\delta_{X}^{\prime}$. Therefore, the $\mathrm{SU}(3)_{F}$ ansatz is justified for nonleptonic charm decays. Consequently, we can proceed with our analysis and study the size of the penguins.

Our fit results to the current data for the relative size of the penguins, or more specifically, the triplet matrix elements which come with $\Delta$, are shown in Fig. 1(b). The generic SM expectation is $\delta_{3}^{(\prime)} \sim P / T \sim 0.1$, while values of $\delta_{3}^{(\prime)} \sim 1$ are generally regarded as an enhancement. As can be seen from Fig. 1(b), the allowed $68 \%$ C.L. region is located at very high values of $\delta_{3}^{(\prime)}$. The border of the $95 \%$ C.L. region can be identified in the zoom of the $\delta_{3}^{\prime}-\delta_{3}$ plane which is shown in Fig. 2(a) and which shows the fit result $\delta_{3}^{(\prime)} \gtrsim \mathscr{O}(1)$. The reason is the following: besides $\Delta a_{C P}^{\mathrm{dir}}$, there are additional $\mathrm{CP}$ asymmetries in the global analysis that are measured with largish central values and generate the need for a penguin enhancement. This is illustrated in Fig. 2(b), where we take for comparison the observables $a_{C P}^{\mathrm{dir}}\left(D^{0} \rightarrow K_{S} K_{S}\right), a_{C P}^{\mathrm{dir}}\left(D_{s} \rightarrow K_{S} \pi^{+}\right)$and $a_{C P}^{\mathrm{dir}}\left(D_{s} \rightarrow K^{+} \pi^{0}\right)$ out of the fit. Without them, also $\delta_{3}^{(\prime)} \lesssim \mathscr{O}(1)$ becomes allowed in the fit. The tendency towards large values for the penguins in the global analysis highlights the importance of future improved measurements of these asymmetries. These will enable us to better constrain the size of the penguins. 


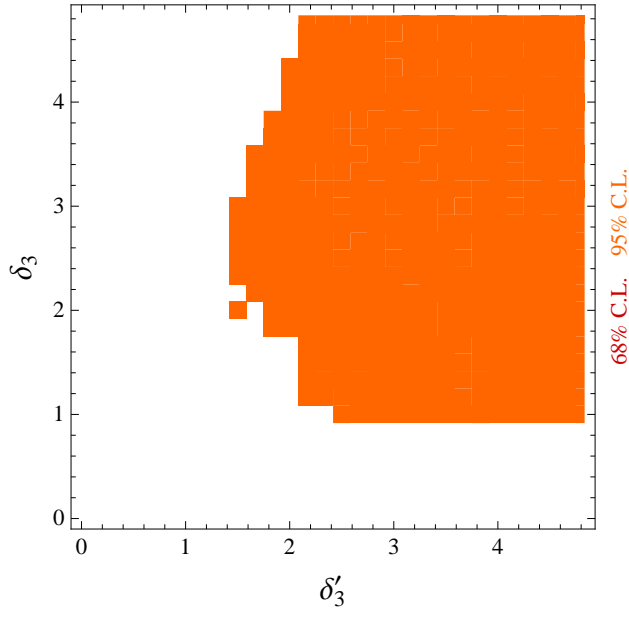

(a) All data.

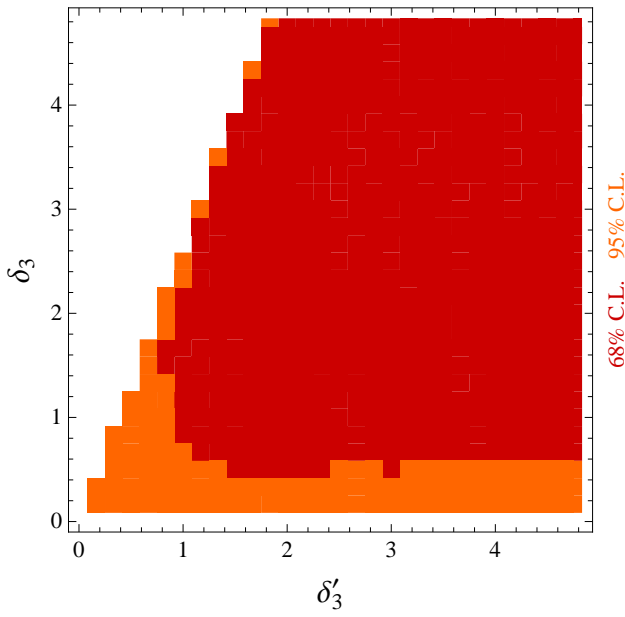

(b) Without large CP asymmetries.

Figure 2: Zoom of fit results in the $\delta_{3}^{\prime}-\delta_{3}$ plane. Left: taking all data into account. Right: Without the CP asymmetries of the decays $D^{0} \rightarrow K_{S} K_{S}, D_{s} \rightarrow K_{S} \pi^{+}$and $D_{s} \rightarrow K^{+} \pi^{0}$. The red (orange) regions are allowed at $68 \%$ and $95 \%$ C.L., respectively, see text for details. Figures taken from [25].

\section{Future data}

There are plans to measure the CP asymmetry $a_{C P}^{\mathrm{dir}}\left(D^{0} \rightarrow \pi^{0} \pi^{0}\right)$ in the future with a $\sim 10$ times smaller uncertainty than is the case at present [20]. We study therefore a hypothetical future data scenario, assuming that $a_{C P}^{\mathrm{dir}}\left(D^{0} \rightarrow \pi^{0} \pi^{0}\right)$ is measured as

$$
a_{C P, \text { future }}^{\text {dir }}\left(D^{0} \rightarrow \pi^{0} \pi^{0}\right)=0.000 \pm 0.006, \quad \text { (future data) }
$$

where the uncertainty is motivated by the prospect given by Belle [20]. The corresponding 95\% C.L. contour of the penguin enhancement differs hardly from the one shown in Fig. 2(a). This result can be understood from counting the degrees of freedom in the fit: in order to determine the two complex penguin matrix elements that induce $\mathrm{CP}$ violation, at least 4 significant measurements of SCS $\mathrm{CP}$ asymmetries are needed. This means that, while present uncertainties leave much room for possible enhancements, even in the pessimistic scenario of no CP violation showing up in $D^{0} \rightarrow \pi^{0} \pi^{0}$ decays, the search for CP violation in SCS decays remains interesting.

\section{Conclusion}

The global $\mathrm{SU}(3)_{F}$ fit of two-body charm decays to kaons and pions shows that an $\mathrm{SU}(3)_{F}$ breaking of $\sim 30 \%$ suffices to describe the data. Furthermore, it reveals a tendency towards large values for the penguins. The penguin enhancement has decreased somewhat with respect to an earlier study [24], especially due to new experimental results on $\Delta a_{C P}^{\mathrm{dir}}$. Nevertheless, taking all observables into account, the overall characteristics found in [24] persist. The observed penguin enhancement is driven, in addition to $\Delta a_{C P}^{\mathrm{dir}}$, by the $\mathrm{CP}$ asymmetries $a_{C P}^{\mathrm{dir}}\left(D^{0} \rightarrow K_{S} K_{S}\right), a_{C P}^{\mathrm{dir}}\left(D_{s} \rightarrow K_{S} \pi^{+}\right)$ and $a_{C P}^{\mathrm{dir}}\left(D_{s} \rightarrow K^{+} \pi^{0}\right)$. These are not measured significantly on their own, so that at present the situation is inconclusive regarding the validity of the Standard Model. It is therefore very important 
to improve these measurements. Furthermore, the tendency to large triplet matrix elements remains assuming a hypothetical but realistic [20] measurement of the CP asymmetry in $D^{0} \rightarrow \pi^{0} \pi^{0}$ at the few-permille level but consistent with zero.

We summarize here the following characteristics of $D \rightarrow P P$ decays, that can serve as a guide for future measurements:

- The CKM-leading contribution to the decay $D^{0} \rightarrow K_{S} K_{S}$ comes first into play when taking $\mathrm{SU}(3)_{F}$ breaking into account. Therefore its $\mathrm{CP}$ asymmetry is enhanced with respect to $\Delta a_{C P}^{\mathrm{dir}}$ as $\mathscr{O}\left(1 / \delta_{X}\right)$ in the SM as well as in generic new physics models $[24,12]$.

- Due to isospin symmetry in the SM to very good precision $a_{C P}^{\mathrm{dir}}\left(D^{+} \rightarrow \pi^{0} \pi^{+}\right)=0$. The violation of this rule would be a smoking gun for $\Delta I=3 / 2$ new physics [24, 39].

- The $\mathscr{O}(1)$ breaking of the $\mathrm{U}$-spin relations

$$
\begin{gathered}
a_{C P}^{\mathrm{dir}}\left(D^{0} \rightarrow K^{+} K^{-}\right)+a_{C P}^{\mathrm{dir}}\left(D^{0} \rightarrow \pi^{+} \pi^{-}\right)=0, \\
a_{C P}^{\mathrm{dir}}\left(D^{+} \rightarrow \bar{K}^{0} K^{+}\right)+a_{C P}^{\mathrm{dir}}\left(D_{s} \rightarrow K^{+} \pi^{0}\right)=0,
\end{gathered}
$$

beyond the amount of $\mathrm{SU}(3)_{F}$ breaking would be a sign of new physics [24]. Especially with regard to Eq. (5.2) there is much space left for an improvement in experimental precision.

- The present tendency towards a penguin enhancement is driven by the CP asymmetries $\Delta a_{C P}^{\mathrm{dir}}$, $a_{C P}^{\mathrm{dir}}\left(D^{0} \rightarrow K_{S} K_{S}\right), a_{C P}^{\mathrm{dir}}\left(D_{s} \rightarrow K_{S} \pi^{+}\right)$and $a_{C P}^{\mathrm{dir}}\left(D_{s} \rightarrow K^{+} \pi^{0}\right)$ [24].

$\mathrm{SU}(3)_{F}$ analysis allows improved $D \rightarrow P P$ measurements to provide more precise information on the borders of the SM and new physics in $|\Delta C|=1$ processes.

\section{Acknowledgments}

StS gratefully acknowledges support by the DFG Research Unit FOR 1873 "Quark Flavour Physics and Effective Field Theories".

\section{References}

[1] R. Aaij et al. [LHCb Collaboration], Phys. Rev. Lett. 108, 111602 (2012) [arXiv:1112.0938 [hep-ex]].

[2] T. Aaltonen et al. [CDF Collaboration], Phys. Rev. Lett. 109, 111801 (2012) [arXiv:1207.2158 [hep-ex]].

[3] D. Pirtskhalava and P. Uttayarat, Phys. Lett. B 712, 81 (2012) [arXiv:1112.5451 [hep-ph]].

[4] T. Feldmann, S. Nandi and A. Soni, JHEP 1206, 007 (2012) [arXiv:1202.3795 [hep-ph]].

[5] J. Brod, Y. Grossman, A. L. Kagan and J. Zupan, JHEP 1210, 161 (2012) [arXiv:1203.6659 [hep-ph]].

[6] J. Brod, A. L. Kagan and J. Zupan, Phys. Rev. D 86, 014023 (2012) [arXiv:1111.5000 [hep-ph]].

[7] H. -Y. Cheng and C. -W. Chiang, Phys. Rev. D 85, 034036 (2012) [arXiv:1201.0785 [hep-ph]].

[8] B. Bhattacharya, M. Gronau and J. L. Rosner, Phys. Rev. D 85, 054014 (2012) [arXiv:1201.2351 [hep-ph]]. 
[9] E. Franco, S. Mishima and L. Silvestrini, JHEP 1205, 140 (2012) [arXiv:1203.3131 [hep-ph]].

[10] G. Isidori, J. F. Kamenik, Z. Ligeti and G. Perez, Phys. Lett. B 711, 46 (2012) [arXiv:1111.4987 [hep-ph]].

[11] Y. Grossman and D. JRobinson, arXiv:1211.3361 [hep-ph].

[12] D. Atwood and A. Soni, PTEP 2013, no. 9, 0903 B05 (2013) [arXiv:1211.1026 [hep-ph]].

[13] G. F. Giudice, G. Isidori and P. Paradisi, JHEP 1204, 060 (2012) [arXiv:1201.6204 [hep-ph]].

[14] G. Hiller, Y. Hochberg and Y. Nir, Phys. Rev. D 85, 116008 (2012) [arXiv:1204.1046 [hep-ph]].

[15] Y. Hochberg and Y. Nir, Phys. Rev. Lett. 108, 261601 (2012) [arXiv:1112.5268 [hep-ph]].

[16] W. Altmannshofer, R. Primulando, C. -T. Yu and F. Yu, JHEP 1204, 049 (2012) [arXiv:1202.2866 [hep-ph]].

[17] G. Isidori and J. F. Kamenik, Phys. Rev. Lett. 109, 171801 (2012) [arXiv:1205.3164 [hep-ph]].

[18] LHCb Collaboration, LHCb-CONF-2013-003, 2013.

[19] RAaij et al. [LHCb Collaboration], Phys. Lett. B 723, 33 (2013) [arXiv:1303.2614 [hep-ex]].

[20] A. J. Schwartz for the Belle Collaboration, Talk at the 6th International Workshop on Charm Physics, 31 August-4 September 2013, Manchester, UK.

[21] Y. Amhis et al. [Heavy Flavor Averaging Group Collaboration], arXiv:1207.1158 [hep-ex].

[22] B. R. Ko [Belle Collaboration], arXiv:1212.1975 [hep-ex].

[23] Y. Grossman, A. L. Kagan and Y. Nir, Phys. Rev. D 75, 036008 (2007) [hep-ph/0609178].

[24] G. Hiller, M. Jung and S. Schacht, Phys. Rev. D 87, 014024 (2013) [arXiv:1211.3734 [hep-ph]].

[25] G. Hiller, M. Jung and S. Schacht, in preparation, DO-TH 13/25, QFET-2013/12, TTP13-037.

[26] S. G. Johnson, http://ab-initio.mit.edu/nlopt.

[27] A.R. Conn, N.I.M. Gould and P. Toint, SIAM J. Numer. Anal. 28,2 (1991) 545-572.

[28] E. G. Birgin and J. M. Martínez, Optimization Methods and Software 23,2 (2008) 177-195.

[29] T. Rowan, PhD thesis, Department of Computer Sciences, University of Texas at Austin, 1990.

[30] B. Aubert et al. [BaBar Collaboration], Phys. Rev. Lett. 100, 061803 (2008) [arXiv:0709.2715 [hep-ex]].

[31] M. Staric et al. [Belle Collaboration], Phys. Lett. B 670, 190 (2008) [arXiv:0807.0148 [hep-ex]].

[32] T. Aaltonen et al. [CDF Collaboration], Phys. Rev. D 85, 012009 (2012) [arXiv:1111.5023 [hep-ex]].

[33] H. Mendez et al. [CLEO Collaboration], Phys. Rev. D 81, 052013 (2010) [arXiv:0906.3198 [hep-ex]].

[34] J. P. Lees et al. [BaBar Collaboration], Phys. Rev. D 87, 052012 (2013) [arXiv:1212.3003 [hep-ex]].

[35] B. R. Ko et al. [Belle Collaboration], Phys. Rev. Lett. 104, 181602 (2010) [arXiv:1001.3202 [hep-ex]].

[36] RAaij et al. [ LHCb Collaboration], JHEP 1306, 112 (2013) [arXiv:1303.4906 [hep-ex]].

[37] A. Zupanc et al. [Belle Collaboration], arXiv:1307.6240 [hep-ex].

[38] P. U. E. Onyisi et al. [ CLEO Collaboration], arXiv:1306.5363 [hep-ex].

[39] Y. Grossman, A. L. Kagan and J. Zupan, Phys. Rev. D 85, 114036 (2012) [arXiv:1204.3557 [hep-ph]]. 